\title{
XXXVI. Krystallographische Untersuchung einiger organischer Substanzen.
}

\author{
II. Reihe.
}

Von

J. Beckenkamp in Mülhausen i. Els.

(Mit 4 Textfiguren.)

1. Phenyl-Akridin, $\mathrm{C}_{6} \mathrm{H}_{4} \cdot C N \cdot C_{6} \mathrm{H}_{5} \cdot \mathrm{C}_{6} \mathrm{H}_{4}$.

Dargestellt von A. Cla us (Journ. f. prakt. Chemie 1892, 46, 126).

Die aus alkoholischer Lösung ausgeschiedenen Krystalle bilden etwa 1-2 mm lange Prismen von hellgelber Farbe (Fig. 1); durch Sublimation erhält man daraus farblose Blättchen von ungefähr gleicher Grösse.

Fig. 1.

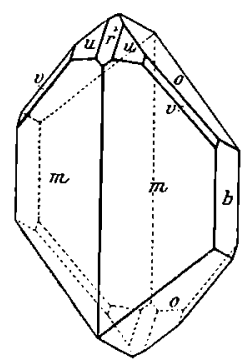

Krystallsystem : Monoklin.

$$
\begin{gathered}
a: b: c=0,9915: 1: 1,0257 ; \\
\beta=27^{\circ} 33^{\prime} .
\end{gathered}
$$

Beobachtete Formen: $m=\{110\} \infty P, o=\left\{T_{11}\right\} P$,

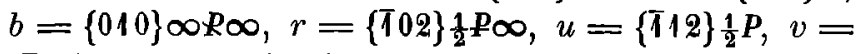
$\left\{\right.$ 1332\} $\frac{3}{2} R 3, a=\{100\} \infty \mathbb{P} \infty$ (Fig. 1).

Bei den aus alkoholischer Lösung gewonnenen Krystallen sind meistens $m$ und $o$ weitaus herrschend oder nur allein vorhanden. Diese wurden deshalb zu Grundformen gewählt, wiewohl dadurch die Axe $a$ so steil wird, dass schon die positiven Formen $r, u$ und $v$ stark nach vorn geneigt sind.

Die durch Sublimation entstehenden Krystalle bilden durch Vorherrschen von $b$ sechsseitige Blättchen, an deren Rändern die ubrigen Flächen als schmale Zuschärfungen auftreten. Durch Umkrystallisiren der farblosen Blättchen aus Alkohol erhält man wieder die gewöhnlichen gelben Krystalle. 
Krystallographische Untersuchung einiger organischer Substanzen. II. Reihe. 573

Es wurde bei

\begin{tabular}{|c|c|}
\hline $\begin{array}{l}\text { den aus Lösung } \\
\text { haltenen Krystallen }\end{array}$ & $\begin{array}{l}\text { den durch Sublimation } \\
\text { erhaltenen Krystallen }\end{array}$ \\
\hline $\begin{array}{l}\text { messen: } \\
9^{\circ} 16^{\prime}\end{array}$ & $\begin{array}{c}\text { gemessen : } \\
49^{0} 23^{\prime}\end{array}$ \\
\hline
\end{tabular}

$m: m=(110):(1 \overline{1} 0)={ }^{*} 49^{0} 16^{\prime} \quad-\quad 49^{\circ} 23^{\prime}$

$m: o=(T 10):(T 11)={ }^{*} 60 \quad 41$

$m: o=($ T10 $):(\bar{T} T 1)=*$ *5 20

$o: o=($ TI1 $):($ TT1 $)=8823$
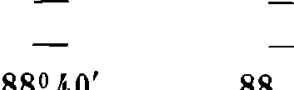

$b: o=(010):(111)=4557$

45,40

8828

$r: u=($ T02 $):($ T12 $)=2152$

2151

$45 \quad 46$

$u: 0=($ T12 $):($ T11) $=51$

516

$o: r=($ T11 $):($ T02 $)=6652$

6639

$m: r=(1 \bar{T} 0):(\overline{1} 02)=5522$

$\begin{array}{ll}55 & 27\end{array}$

$u: v=(\overline{1} 12):(\overline{1} 32)=2810$

2824

$28 \quad 15$

$v: b=($ T32) $:(010)=3938$

$\begin{array}{ll}39 & 45\end{array}$

$v: 0=(\bar{T} 32):(\bar{T} 11)=$

$37 \quad 44$

2141

Auf $m$ beträgt die Schiefe der Auslöschung $6^{\circ}$ gegen die $c$-Axe, auf $b$ $16^{0}$ gegen dieselbe Richtung. Die aus Lösung erbaltenen gelben Kryställchen sind pleochroitisch; geht die $c$-Axe ungefähr parallel der kurzen Diagonale des Nicols, so erscheinen sie (auf $m$ liegend) gelb, geht die $c$-Axe parallel der langen Nicoldiagonalen, so sind sie farblos. Die durch Sublimation entstehenden Blättchen sind in allen Lagen farblos durchsichtig; dass aber trotzdem beide Bildungsweisen identische Formen liefern, ist nach der mitgetheilten Tabelle unzweifelhaft.

Auch aus Chloroform erhält man das Phenylakridin in Krystallen von der gleichen gelben Farbung, die jedoch Krystallchloroform enthalten und sehr rasch verwittern.

Die Identität der farblosen und gefärbten Krystalle beweist, dass die Färbung nur durch intramolekular eingeschlossene Substanzen veranlasst sein kann; und da die Färbung bei verschiedenen Lösungsmitteln dieselbe ist, so können die eingeschlossenen Substanzen nicht aus dem Lösungsmittel stammen, sondern es sind dissociirte Krystallmoleküle.

\section{Salpetersaures Jodosobenzol, $\mathrm{C}_{6} H_{5} . J\left(O_{3} N\right)_{2}$.}

Dargestellt von C. Willgerodt (Ber. d. d. chem. Ges. 1893, 26, 1307).

Krystallsystem: Monoklin.

$$
\begin{gathered}
a: b: c=2,3124: 1: 1,2784 ; \\
\beta=51045^{\prime} .
\end{gathered}
$$

Die Krystalle bilden dicke, gelbe Tafeln nach $\{001\} 0 P$. Ausser dieser Fläche sind stets die Flächen $\{100\} \infty P \infty$ und $\{110\} \infty P$ vorhanden; $\{T 11\}+P$ wurde nur einmal als schmale Abstumpfung beobachtet. Dem- 
entsprechend ist der Werth der $c$-Axe auch weniger genau als der der anderen Axenelemente. Es wurden

\begin{tabular}{|c|c|c|}
\hline \multicolumn{2}{|c|}{ Beobachtet: } & \multirow{2}{*}{$\begin{array}{c}\text { Berechnet } \\
-\end{array}$} \\
\hline$(100):(001)=5$ & $51045^{\prime}$ & \\
\hline$(100):(110)=6$ & 612 & - \\
\hline$(001):(T 11)=5$ & 5853 & - \\
\hline$(110):(001)=7$ & 7225 & $72033^{\prime}$ \\
\hline$(\bar{T} T 1):(\bar{T} 11)=10$ & 0322 & 10329 \\
\hline$(\bar{T} \mid \mathbb{1}):(\bar{T} \mid 0)=4$ & $48 \quad 42$ & $48 \quad 42$ \\
\hline$(100):(T 11)=8$ & 8743 & $87 \quad 2$ \\
\hline
\end{tabular}

Die Krystalle sind nur scbwach pleochroitisch; die Auslöschung auf (100) beträgt $35^{0}$ gegen die Verticale.

3. Guajacol, $\mathrm{C}_{6} \mathrm{H}_{4}(\mathrm{OH})\left(\mathrm{OCH}_{3}\right)$.

Dargestellt von den Fabriques de produits chimiques de Thann et de Mulhouse (vergl. G. Freys, Chem.-Ztg. 1894, 18, 19).

Krystallsystem: Hexagonal, trapezoëdrisch-tetartoëdrisch.

$$
a: c=1: 0,9933 \text {. }
$$

Fig. 2.

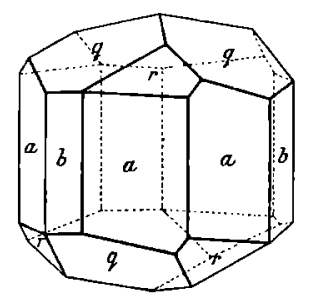

Beobachtete Formen (Fig. 2): $a=\{10 \pi 0\} \infty R$, $b=\{2 \bar{T} \bar{T} 0\} \frac{\infty P 2}{4} l, b^{\prime}=\{11 \overline{2} 0\} \frac{\infty P 2}{4} r, r=\{10 \bar{T} 1\} R$, $q=\{2 T T 3\} \frac{\frac{2}{3} P 2}{4} l$.

Die Krystalle sind häufig mehrere Centimeter lang und etwa halb so dick, anfangs farblos, aber dem Sonnenlichte ausgesetzt färben sie sich schwach rosa; sie besitzen einen starken, eigenthumlichen Geruch. Das Prisma erster Ordnung $a$ tritt immer mit sechs Flächen auf und ist meist die herrschende Form. Das Prisma zweiter Ordnung $b$ ist băufig nur als linkes trigonales vorhanden; wenn auch das rechte trigonale Prisma $b^{\prime}$ auftritt, so ist es zuweilen im Gegensatze zu dem linken matt und schmal. Von der Pyramide zweiter Ordnung wurde nur die linke trigonale Form $q$ als schiefe Abstumpfung der Polkanten des Rhomboëders $r$ beobachtet.

$$
\begin{aligned}
& \text { Beobachtet: Berechnet: } \\
& r: r=(10 T 1):(0 \pi 11)=81030^{\prime} \text {. } \\
& r: q=(10 T 1):(2 T T 3)=2510 \\
& q: r=(2 \pi T 3):(0 T 11)=5620 \quad 5646 \\
& r: a=(10 \pi 1):(10 \pi 0)=4121 \quad 415 \\
& r: a=(10 \pi 1):(01 \pi 0)=-6751
\end{aligned}
$$




$$
\begin{array}{lc}
\text { Beobachtet: } & \text { Berechnet: } \\
r: b^{\prime}=(10 \bar{T} 1):(1 \overline{2} 0)=49022^{\prime} & 49015^{\prime} \\
a: q=(10 \bar{T} 0):(2 \bar{T} \bar{T})=- & 6122 \\
q: b=(2 \bar{T} \bar{T}):(2 \bar{T} 0)=5615 & 5630
\end{array}
$$

Oplisch positiv, nicht activ, $\omega_{N a}=1,569, \varepsilon_{N a}=1,666$.

Der niedrige Schmelzpunkt $\left(27{ }^{\circ} \mathrm{C}\right.$.) ist für die geometrische und optische Bestimmung recht ungünstig, da die Bilder schon nach wenigen Messungen verschwommen und unbrauchbar werden.

\section{Diacetyldiamidodibrombenzol,}

$$
\mathrm{C}_{6} \mathrm{H}_{2}\left(\mathrm{NHCOCH}_{3}\right)_{2} \stackrel{(2,6)}{\mathrm{Br}_{2}} \text {. }
$$

Dargestellt und gemessen von O. Mich el (Inaug.-Dissert. d. Univ. Basel).

Krystallsystem : Rhombisch.

$$
a: b: c=0,6793: 1: 1,4451 \text {. }
$$

Fig. 3.

Beobachtete Formen: $c=\{001\} 0 P, o=$

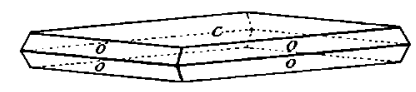
$\{111\} P$ (Fig. 3).

Die Krystalle bilden dünne, farblose Blättchen nach der Basis.

Beobachtet: Berechnet:

$$
\begin{aligned}
& c: 0=(001):(111)=68^{0} 45^{\prime} \\
& o: o=(111):(1 \overline{1} 1)=6310 \\
& o: 0=(111):(\overline{1} 11)=10020 \quad 100^{\circ} 53^{\prime}
\end{aligned}
$$

\section{Acetylparanitrophenol,}

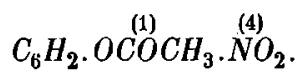

Dargestellt und gemessen von O. Michel (ebenda).

Krystallsystem: Rhombisch.

$$
a: b: c=0,9204: 1: 0,8826 .
$$

Beobachtete Formen: $c=\{001\} 0 P, m=\{110\} \infty P, r=\{101\} \bar{P} \infty$.

Farblose, nach der Basis tafelförmige Krystalle.

$$
\begin{array}{llc} 
& \text { Beobachtet: } & \text { Berechnet: } \\
m: m=(110):(110)=85^{0} 5^{\prime} & - \\
c: r=(001):(101)=4348 & - \\
r: m=(101):(110)=5942 & 59^{0} \mathbf{2 3}^{\prime}
\end{array}
$$




\section{Acetylorthodibromparanitroanilin,}

$$
\mathrm{C}_{6} \mathrm{H}_{2} \cdot \mathrm{NHCOCH}_{3} \cdot \stackrel{(4)}{\mathrm{NO}_{2}} \stackrel{\stackrel{(2,6)}{\mathrm{B}} r_{2}}{\text {. }}
$$

Dargestellt und gemessen von 0 . Michel (ebenda).

Fig. 4.

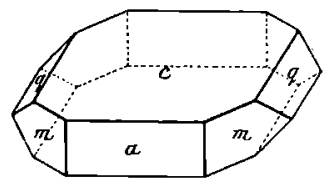

Krystallsystem: Monoklin.

$$
\begin{gathered}
a: b: c=0,8161: 1: 0,9566 ; \\
\beta=71^{\circ} 0^{\prime} .
\end{gathered}
$$

Beobachtete Formen: $c=\{001\} 0 P, a=\{100\}$ $\infty P \infty, m=\{110\} \infty P, q=\{011\}$ R $\infty$ (Fig. 4).

Nach der Basis tafelförmige, theils gleichseitige, theils nacb der $b$-Axe verlängerte Krystalle.

$$
\begin{aligned}
& \text { Beobachtet: Berechnet: } \\
& c: a=(001):(100)=7100^{\prime} \\
& q: c=(011):(001)=4440 \\
& a: m=(100):(110)=3739 \\
& a: q=(100):(011)=77 \quad 0 \\
& 76^{0} 37^{\prime} \\
& c: m=(001):(110)=7630 \\
& 754
\end{aligned}
$$

\section{Metanitrometaxylolazoimid,}

$$
\mathrm{C}_{6} \mathrm{H}_{2} \cdot \stackrel{(1)}{\mathrm{N}_{3}} \cdot \stackrel{(3)}{\mathrm{NO}_{2}} \cdot\left(\stackrel{(4,6)}{\mathrm{C}} \mathrm{H}_{3}\right)_{2} \text {. }
$$

Dargestellt und gemessen von 0 . Michel (ebenda).

Krystallsystem : Monoklin.

$$
\begin{gathered}
a: b: c=1,2453: 1: ? ; \\
\beta=78^{\circ} 51^{\prime} .
\end{gathered}
$$

Beobachtete Formen: $c=\{001\} 0 P, a=\{100\} \infty P_{\infty}, m=\{110\} \infty P$. Braune Blättchen nach der Basis.

$$
\begin{array}{lcc}
a: m=(100):(110)=50^{0} 42^{\prime} & \text { Beobachtet } & - \\
c: m=(001):(110)=8258 & - \\
a: c=(100):(001)=7830 & 78^{0} 51^{\prime}
\end{array}
$$

Die Auslöschungsschiefe auf (110) beträgt $7^{0}$ gegen die Verticalaxe.

Auf der Basis erscheint der nach (Fresnel) parallel $b$ schwingende Strahl braunroth, der parallel $a$ schwingende braungelb. 